\title{
EFECTO DEL ESTRONCIO EN LAS TEMPERATURAS DE REACCION EUTECTICAS Y MICROESTRUCTURAS DE SOLIDIFICACION DE UNA ALEACION Al-Si TIPO A319
}

\author{
$\begin{array}{lll}\text { E. Martínez } & \text { J. Lacaze } & \text { M. Cisneros }\end{array}$ \\ Recibido el 14 de julio de 2004, aceptado el 30 de octubre de 2004
}

\begin{abstract}
RESUMEN
El presente trabajo se realizó sobre una aleación base Al-Si-Cu, muy utilizada en el sector automotriz para la fabricación de monoblocks y cabezas de motor. Se utilizó el análisis térmico diferencial (DTA) para determinar las temperaturas de inicio y fin de las reacciones que ocurren durante la solidificación de la aleación A319 a tres velocidades de enfriamiento $\left(2,10\right.$ y $\left.20{ }^{\circ} \mathrm{C} / \mathrm{min}\right)$ con 12 y $136 \mathrm{ppm}$ de $\mathrm{Sr}$ como agente modificador. Se detectaron tres reacciones eutécticas, la más interesante entre ellas es la formación de fases ricas en cobre, con temperaturas de formación que disminuyen al aumentar la velocidad de enfriamiento y el contenido de Sr. Finalmente, se efectuó un microanálisis empleando microscopio electrónico de barrido para determinar la distribución de los elementos en las fases obtenidas.
\end{abstract}

Palabras claves: Estroncio, análisis térmico diferencial, aleación de Aluminio A319, Reacción eutéctica.

\begin{abstract}
This work was carried out over a basic Al-Si-Cu alloy, widely used in the automotive industry for production of monoblocks and motor heads. Differential thermal analysis (DTA) was used to determine the reaction temperatures from beginning to end of the solidification process of alloy type $\mathrm{A} 319$, using three cooling rates $\left(2,10\right.$, and $\left.20^{\circ} \mathrm{C} / \mathrm{min}\right)$ on samples with 12 and 136 ppm of Sr as modifying agent. Three eutectic reactions were found, one of these reactions corresponds to eutectic reaction of phases rich in copper and it was also found that the reaction temperature decreases when the cooling rate increases and also when the strontium content increases. Microanalysis using electron microscopy sacnning was performed, to determine the element distribution in the resulting phases.
\end{abstract}

Keywords: Strontium, differential thermal analysis, Aluminum alloy A319, Eutectic reaction.

\section{INTRODUCCIÓN}

Las aleaciones $\mathrm{Al}-\mathrm{Si}$ son ampliamente usadas en la industria automotriz, estructuras aerospaciales y aplicaciones militares, esto debido a sus buenas propiedades mecánicas, bajo peso, buena soldabilidad, buena conductibilidad térmica, relativamente alta resistencia a alta temperatura, excelente resistencia a la corrosión, así como excelente fluidez al vaciado [1]. Debido a la evolución de las microestructuras durante el proceso de solidificación, la microestructura no solamente determina las propiedades mecánicas, si no que también define los limites del diseño de vaciado, la colabilidad de la aleación y la susceptibilidad a la formación de defectos [2]. La forma del Si eutéctico juega un papel muy importante, la cual se modifica mediante la adición de $\mathrm{Sr}$ al baño metálico, cambiando la morfología del eutéctico de acicular a fibrosa, y con ello mejorando los propiedades mecánicas de la aleación [3]. El efecto de la velocidad de enfriamiento en el análisis térmico de aleaciones tratadas con $\mathrm{Sr}$ no ha sido extensamente estudiado y solo se conoce el efecto del $\mathrm{Sr}$ sobre el Si y no se sabe su efecto sobre otros productos de reacción [4]. Backerud y colaboradores [5] reportaron la secuencia de solidificación para la aleación 319 en cinco reacciones, donde mencionan que la primera reacción corresponde a la precipitación del $\alpha \mathrm{Al}$ a $609{ }^{\circ} \mathrm{C}$, la segunda reacción se presenta a $590{ }^{\circ} \mathrm{C}$ presentándose en forma simultánea las reacciones $\mathrm{L} \rightarrow$ $\alpha \mathrm{Al}+\mathrm{Al}_{15} \mathrm{Mn}_{3} \mathrm{Si}_{2}$ y L $\rightarrow \alpha \mathrm{Al}+\mathrm{Al}_{5} \mathrm{FeSi}+\mathrm{Al}_{15} \mathrm{Mn}_{3} \mathrm{Si}_{2}$, una tercera reacción a $575{ }^{\circ} \mathrm{C}$ correspondiente a $\mathrm{L} \rightarrow$ $\alpha \mathrm{Al}+\mathrm{Si}+\mathrm{Al}_{5} \mathrm{FeSi}$, a $525{ }^{\circ} \mathrm{C}$ reportan la cuarta reacción $\mathrm{L} \rightarrow \alpha \mathrm{Al}+\mathrm{Al}_{2} \mathrm{Cu}+\mathrm{Si}+\mathrm{Al}_{5} \mathrm{FeSi}$ y finalmente a $507^{\circ} \mathrm{C}$ la reaccion $\mathrm{L} \rightarrow \alpha \mathrm{Al}+\mathrm{Al}_{2} \mathrm{Cu}+\mathrm{Si}+\mathrm{Al}_{5} \mathrm{Mg}_{6} \mathrm{Cu}_{2} \mathrm{Si}_{6}$. Apelian y colaboradores [6] reportaron la formación de dos morfologías distintas del precipitado $\mathrm{Al}_{2} \mathrm{Cu}$ durante

\footnotetext{
${ }^{1}$ Instituto Tecnológico de Zacatecas México, aspirante al grado de doctor, enriquemartinez_1999@yahoo.com

${ }^{2}$ INP-ENSIACET, CIRIMAT, Toulouse Francia

${ }^{3}$ Instituto Tecnológico de Saltillo. Saltillo Coah. México

${ }^{4}$ Nemak S.A. de C.V., R \& D, Monterrey N.L. México
} 
la solidificación de la aleación 319, estas son: 1.morfología Blocky (Block-like) conteniendo $40 \%$ de $\mathrm{Cu}$ y 2.- una fase eutéctica fina la cual contiene aproximadamente $24 \%$ de $\mathrm{Cu}$. Samuel y Doty [7] reportan un análisis microestructural de una aleación 319 vaciada en un molde con templadera (chill), con alrededor de $300 \mathrm{ppm}$ de $\mathrm{Sr}$, estudiando también el efecto de la refinación de grano, variando el contenido de $\mathrm{Fe}$ y $\mathrm{Mg}$, así mismo realizaron Análisis Térmico (TA) y Calorimetría diferencial por barrido (DSC) encontrando en su estudio 4 reacciones eutécticas, reportan que cuando la aleación se enfría rápidamente la fase $\mathrm{Al}_{2} \mathrm{Cu}$ se presenta principalmente en forma de racimo (pocket) de eutéctico fino $\left(\mathrm{Al}_{2} \mathrm{Cu}\right)$ en las regiones interdendríticas junto con partículas de $\mathrm{Si}$, también sugieren que la precipitación de las fases ricas en $\mathrm{Cu}$, primero se precipita en forma Blocky y a partir de este en forma eutéctico fino en el rango de temperaturas de 510 a $490{ }^{\circ} \mathrm{C}$. En este trabajo se aplicó el análisis térmico diferencial (DTA), el cual no ha sido aplicado a este tipo de Aleaciones y así mismo un estudio microestructural para contribuir a una mejor comprensión la secuencia de solidificación de las fases formadas en una aleación elaborada por la industria automotriz.

\section{EXPERIMENTACIÓN}

\section{Elaboración de la Aleación}

El metal fue procesado en un horno rotatorio de 11 toneladas cuya carga fue seleccionada en base a chatarra clasificada. Posteriormente el metal líquido fue tratado en ollas de 1 tonelada para ser modificada con aleaciones maestras $\mathrm{Al}-\mathrm{Sr}$, controlando la cantidad de estroncio, el metal líquido fue desgasificado mediante la inyección de nitrógeno y refinado con el Ti residual de la chatarra. La temperatura de vaciado fue de $760{ }^{\circ} \mathrm{C}$, la Fig. 1 muestra la pieza obtenida, con este diseño se permite una alimentación continua para evitar la formación de defectos. Las probetas posteriormente fueron maquinadas en forma de barras con dimensiones finales de $6 \mathrm{~mm}$ de diámetro y $100 \mathrm{~mm}$ de longitud. La Tabla 1 muestra la composición química de las muestras base.

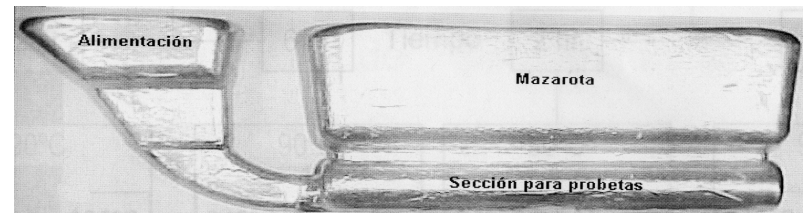

Fig. 1 Fotografía mostrando la sección de donde se obtuvieron las probetas.
Tabla 1. Composición química de la aleación utilizada

\begin{tabular}{|c|c|c|c|c|c|c|c|}
\hline & $\begin{array}{c}\mathrm{Si} \\
(\% \mathrm{wt})\end{array}$ & $\begin{array}{c}\mathrm{Cu} \\
(\% \mathrm{wt})\end{array}$ & $\begin{array}{c}\mathrm{Fe} \\
(\% \mathrm{wt})\end{array}$ & $\begin{array}{c}\mathrm{Mn} \\
(\% \mathrm{wt})\end{array}$ & $\begin{array}{c}\mathrm{Mg} \\
(\% \mathrm{wt})\end{array}$ & $\begin{array}{c}\mathrm{Ti} \\
(\% \mathrm{wt})\end{array}$ & $\begin{array}{c}\mathrm{Sr} \\
(\mathrm{ppm})\end{array}$ \\
\hline $\mathbf{A}$ & 7.23 & 3.33 & 0.621 & 0.422 & 0.295 & 0.081 & 136 \\
\hline $\mathbf{B}$ & 7.31 & 3.27 & 0.642 & 0.424 & 0.285 & 0.073 & 12 \\
\hline
\end{tabular}

$\mathrm{Al}$ inicio de las pruebas se aplicó vacío en la cámara del horno seguido de un llenado con Ar con un flujo de 2 lts/min, de tal modo que las muestras fueron fundidas bajo una atmósfera de argón. La Tabla 2 muestra la nomenclatura utilizada.

Tabla 2. Condiciones de la experimentación y nomenclatura

\begin{tabular}{|c|c|c|c|c|c|}
\hline \multicolumn{2}{|c|}{$20^{\circ} \mathrm{C} / \mathrm{min}$} & \multicolumn{2}{c|}{$10{ }^{\circ} \mathrm{C} / \mathrm{min}$} & \multicolumn{2}{c|}{$2{ }^{\circ} \mathrm{C} / \mathrm{min}$} \\
\hline Alto Sr & Bajo Sr & Alto Sr & Bajo Sr & Alto Sr & Bajo Sr \\
\hline A1 & B1 & A2 & B2 & A3 & B3 \\
\hline
\end{tabular}

\section{Análisis Microestructural}

Las muestras obtenidas del DTA fueron cortadas longitudinalmente, después montadas y preparadas metalográficamente, estas no fueron atacadas, solo se pulieron en su última etapa con pasta de alúmina de 1 $\mu \mathrm{m}$. Se utilizó un microscopio óptico de platina invertida marca OLIMPUS PMG3. Igualmente se realizo microanálisis por EDS (IMIX-PC-PGT) con detector de $\mathrm{Ge}$, en un microscopio electrónico de barrido marca LEO modelo 435 VP.

\section{RESULTADOS Y DISCUSIÓN}

\section{Análisis Térmico Diferencial}

Las curvas obtenidas para ambas aleaciones fueron comparadas para cada velocidad de enfriamiento en la Fig. 2. Mediante el cambio de pendiente en los puntos de inflexión de cada curva se determinaron las temperaturas de inicio y final de reacción obtenidas de este análisis con un factor de error de 0.009 [9], las cuales se muestran en la Tabla 3, la variación de estas curvas se debe al desprendimiento del calor de transformación y se determinan mediante la primera derivada de la temperatura con respecto al tiempo. La penúltima reacción solo se detectó a la velocidad de enfriamiento de $2{ }^{\circ} \mathrm{C} / \mathrm{min}$, para ambos contenidos de estroncio. 

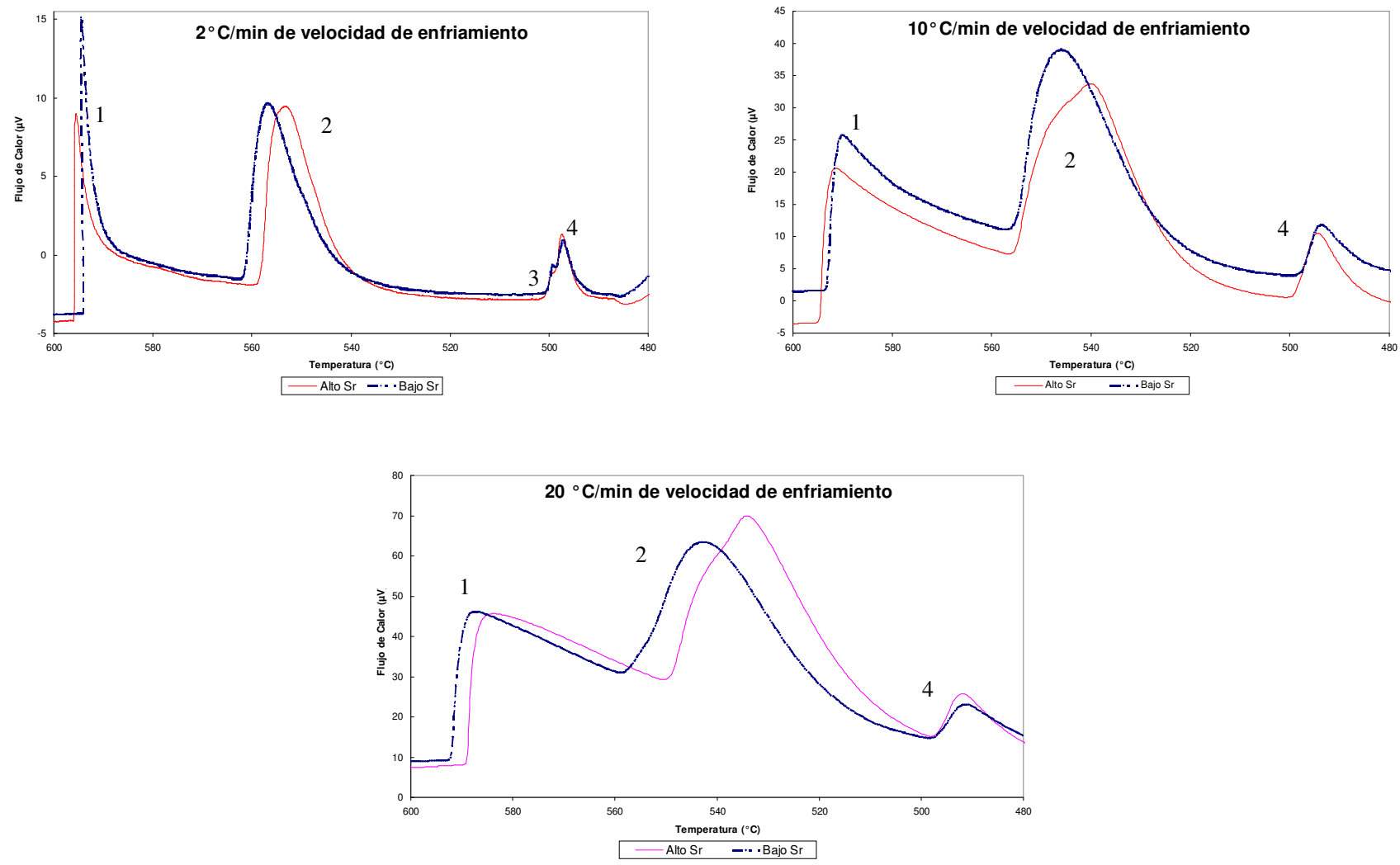

Fig. 2.- Efecto del Sr sobre las reacciones eutécticas a las velocidades de enfriamiento estudiadas

Tabla 3. Temperaturas de inicio y final de reacción, obtenidas en el análisis DTA. (Error $\pm 0.01^{\circ} \mathrm{C}$ )

\begin{tabular}{|c|c|c|c|c|c|c|}
\hline & \multicolumn{6}{|c|}{ Temperatura Inicio de Reacción $\left({ }^{\circ} \mathrm{C}\right)}$. \\
\hline Reacción & A1 & B1 & A2 & B2 & A3 & B3 \\
\hline 1 & 589,3 & 592,2 & 594,6 & 593,2 & 596,0 & 594,5 \\
\hline 2 & 549,8 & 558,3 & 555,5 & 555,4 & 558,3 & 556,8 \\
\hline 3 & ------- & ------- & ------- & ------- & 500,8 & 500,6 \\
\hline 4 & 497,8 & 497,8 & 499,7 & 497,8 & 498,8 & 498,3 \\
\hline & \multicolumn{7}{|c|}{ Temperatura Final de Reacción $\left({ }^{\circ}\right.$ C) } \\
\hline Reacción & A1 & $\begin{array}{r}\text { B } \\
1\end{array}$ & A2 & B2 & A3 & B3 \\
\hline 1 & 584,3 & 587,4 & 591,8 & 590,3 & 595,6 & 593,0 \\
\hline 2 & 534,0 & 542,9 & 540,0 & 546,4 & 553,2 & 555,8 \\
\hline 3 & ------- & ------- & ------- & ------- & 499,2 & 499,3 \\
\hline 4 & 492,1 & 491,8 & 494,5 & 494,1 & 497,5 & 497,1 \\
\hline
\end{tabular}

Las reacciones detectadas en este análisis corresponden, la primera a la formación de los granos de $\alpha$-Al que inician su precipitación entre 594.6 a $589.6{ }^{\circ} \mathrm{C}$ para las muestras con alto contenido de estroncio, mientras que para las de bajo contenido de estroncio entre $594.5^{\circ} \mathrm{C}$ a $592.2{ }^{\circ} \mathrm{C}$, las diferencias con la bibliografía consultadas se deben a la diferencia en contenido de elementos menores de aleación[5]. La segunda inflexión corresponde a la precipitación del eutéctico principal $\alpha \mathrm{Al}+\mathrm{Si}$, de acuerdo a la reacción $\mathrm{L} \rightarrow \alpha \mathrm{Al}+\mathrm{Si}$ entre los 558.3 a $549.8{ }^{\circ} \mathrm{C}$ para las muestras con alto estroncio y entre 558.3 a $555.4{ }^{\circ} \mathrm{C}$ para bajo contenido de estroncio [5], [6]. La tercera reacción solo se detectó a 2 ${ }^{\circ} \mathrm{C} /$ min de enfriamiento en ambos contenidos de $\mathrm{Sr}$ y corresponde a la formación de la fase $\mathrm{Al}_{2} \mathrm{Cu}$ con morfología de eutéctico fino, esta solo se detectó a $2{ }^{\circ} \mathrm{C} / \mathrm{min}$, a las otras dos velocidades de enfriamiento no se detectó, ya que a altas velocidades de enfriamiento se desfavorece la morfología de eutéctico fino del $\mathrm{Al}_{2} \mathrm{Cu}$ [6]. Finalmente la reacción cuatro que se detectó entre 499.7 a $497.8{ }^{\circ} \mathrm{C}$ para alto contenido de estroncio y 498.3 a $497.8{ }^{\circ} \mathrm{C}$ para bajo contenido de estroncio, dicha reacción corresponde a la precipitación de las multifases, $\alpha \mathrm{Al}+\mathrm{Al}_{2} \mathrm{Cu}+\mathrm{Si}+$ $\mathrm{Al}_{5} \mathrm{Mg}_{6} \mathrm{Cu}_{2} \mathrm{Si}_{6}$ [5], [6]. Se observa que, al aumentar la velocidad de enfriamiento disminuye la temperatura de la 
reacción, lo anterior se debe a que conforme aumenta la velocidad de enfriamiento, el proceso de solidificación se aleja cada vez más del equilibrio [8]. A la velocidad de enfriamiento de $2{ }^{\circ} \mathrm{C} / \mathrm{min}$, solo se detectó un ligero efecto del $\mathrm{Sr}$ sobre la reacción 1 y 2 , la primera la aumenta alrededor de $2{ }^{\circ} \mathrm{C}$, mientras que a la segunda, la disminuye alrededor de $4{ }^{\circ} \mathrm{C}$. Para la velocidad de 10 ${ }^{\circ} \mathrm{C} / \mathrm{min}$, solo se observa un leve efecto del $\mathrm{Sr}$ sobre la temperatura de las reacciones 1 y 3, aumentándolas reacciones 1 y 2 en forma más marcada que las

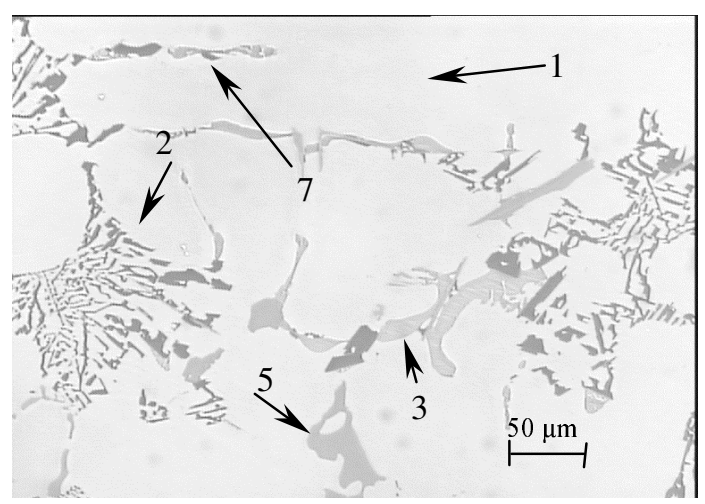

a). Muestra A1

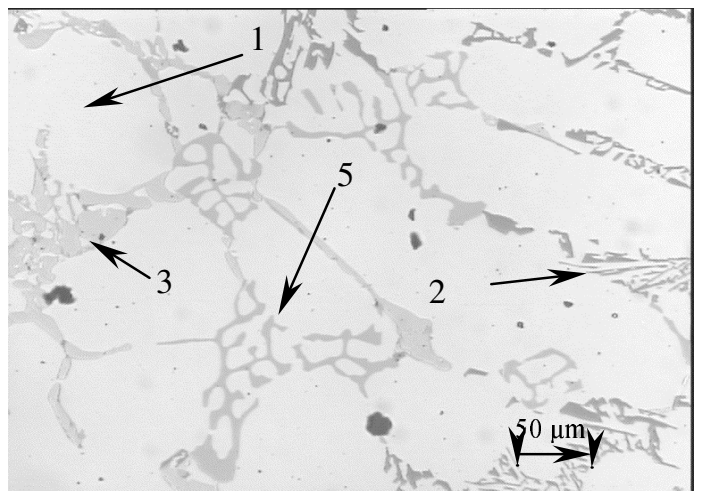

c). Muestra A1

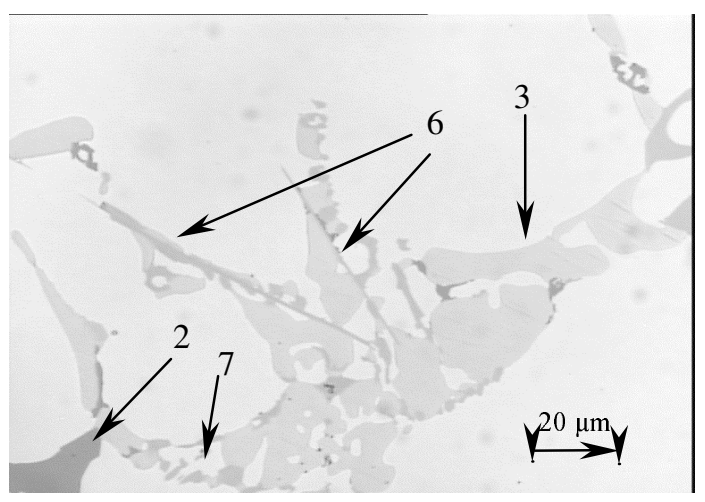

e) Muestra A1 anteriores, de alrededor de 3 y $9{ }^{\circ} \mathrm{C}$ respectivamente, en forma general, podemos decir que el estroncio tiene un efecto significativo a velocidades de enfriamiento altas, disminuyendo principalmente la temperatura del eutéctico principal $\alpha \mathrm{Al}+\mathrm{Si}$, este mismo comportamiento es reportado por otros investigadores [3], [4] y [7]. Closset \& Gruzleski [3], [4] sugieren que el Sr modifica la morfología de la interfase sólido-líquido, pero a bajas velocidades de enfriamiento se inhibe el efecto del Sr.

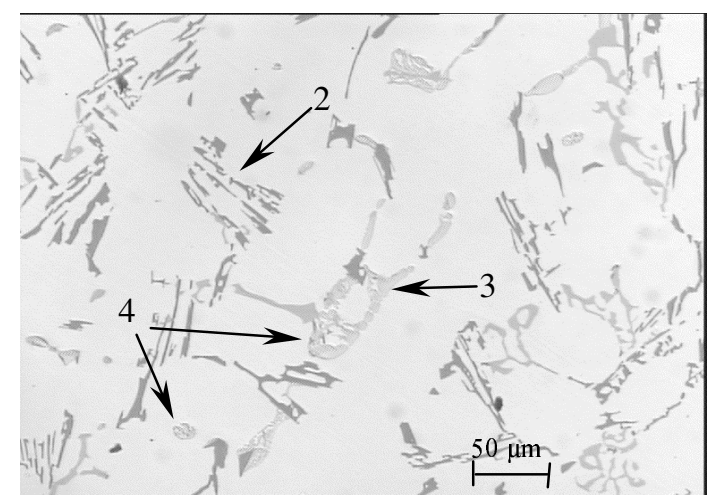

b). Muestra B1

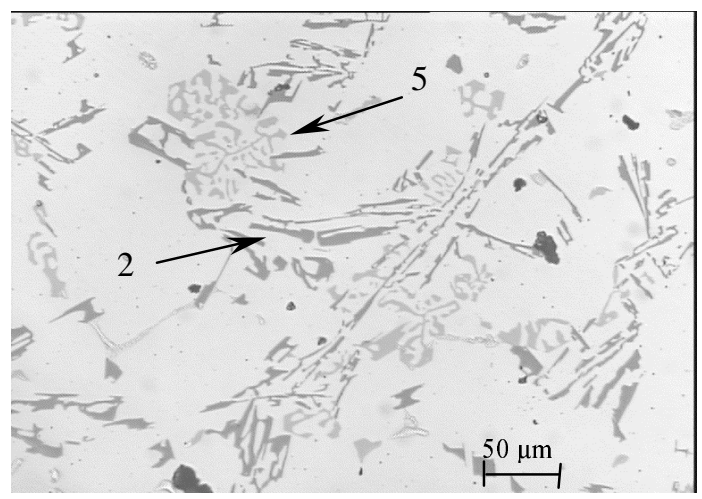

d). Muestra B 1

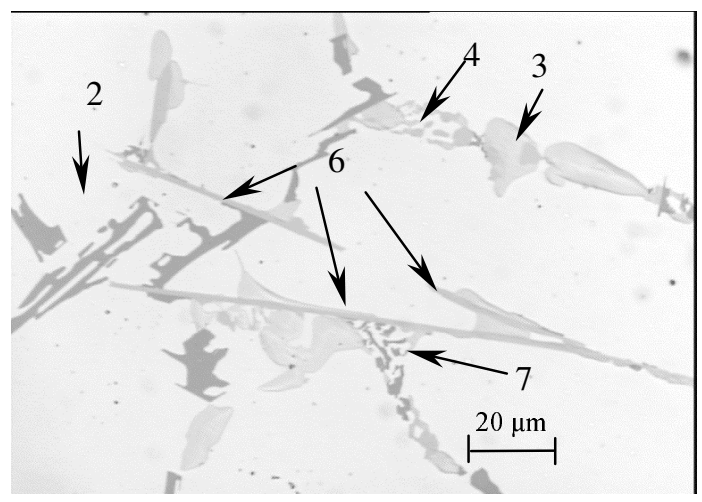

f) Muestra B1 


\section{Análisis Microestructural}

Las microestructuras obtenidas a $20{ }^{\circ} \mathrm{C} /$ min con alto y bajo estroncio, se muestran en la figura 3 . En forma general se observaron 7 fases en todas las muestras, las cuales varían tanto en cantidad como en forma de acuerdo a la velocidad de enfriamiento y al contenido de estroncio. En forma general se observaron 7 fases en todas las muestras, las cuales varían tanto en cantidad como en forma de acuerdo a la velocidad de enfriamiento y al contenido de estroncio. El análisis cualitativo de las muestras mostró que las fases ricas en $\mathrm{Cu}$ solidifican en dos tipos diferentes de morfología, en tipo "Blocky" y en forma de eutéctico fino, se pudo apreciar que en la muestra con alto contenido de estroncio se favorece la formación de $\mathrm{Al}_{2} \mathrm{Cu}$ en forma "Blocky" hacia las zonas interdendríticas. En relación a las fases en forma de aguja $\beta-\mathrm{Al}_{5} \mathrm{FeSi}$, se observan mas finas de menor longitud y distribuidas en toda la muestra cuando el contenido de estroncio es alto, en cambio para las muestras con bajo contenido de estroncio, estas agujas son mas alargadas, mas numerosas y bastas, que en comparación con alto contenido de estroncio; en cuanto al precipitado en forma de escritura china, $\alpha-\mathrm{Al}_{15}(\mathrm{Fe}, \mathrm{Mn})_{3} \mathrm{Si}_{2}$, el estroncio favorece su formación y engrosamiento, ya que se aprecio mas fino y en menor cantidad que en las muestras con bajo estroncio, esto seguramente es debido al efecto de Sr sobre la interfase sólido - líquido como lo reporta Closset y Gruzleski [4]. Aparentemente la velocidad de enfriamiento juega un papel muy importante en la morfología y cantidad de segundas fases presentes ya que solo a velocidades de enfriamiento altas $\left(10\right.$ y $\left.20^{\circ} \mathrm{C} / \mathrm{min}\right)$ se puede detectar la presencia del eutéctico fino $\mathrm{Al}_{2} \mathrm{Cu}$ tanto en las muestras con alto y bajo estroncio [6]. Mediante el análisis de las microestructuras, el eutéctico fino rico en $\mathrm{Cu}$ esta ausente a velocidades de enfriamiento de $2{ }^{\circ} \mathrm{C} / \mathrm{min}$ en las muestras con alto y bajo contenido de $\mathrm{Sr}$, esto se debe a que el aumento de la velocidad de enfriamiento y Sr, favorece la morfología tipo "blocky" de las fases ricas en $\mathrm{Cu}$ [4], [6]. Se observó que el efecto del $\mathrm{Sr}$ disminuye con el aumento en la velocidad de enfriamiento, ya que las microestructuras en las muestras con alto y bajo contenido de estroncio a 2 ${ }^{\circ} \mathrm{C} / \mathrm{min}$, presentan estructuras muy similares [4]. Se puede apreciar de acuerdo a la distribución y cercanía de los diferentes precipitados que las fases en forma de escritura china, nuclean en los límites del silicio eutéctico, a su vez estos sirven de nucleantes del $\mathrm{Al}_{2} \mathrm{Cu}$ en forma "Blocky" y este se descompone en el eutéctico $\mathrm{Al}_{2} \mathrm{Cu}$, no obstante parece ser que los precipitados en forma de aguja sirven también como sitios de nucleación de las fases ricas en $\mathrm{Cu}$ [6].
Se realizó un análisis químico cualitativo por mapeo mediante EDS, en la muestra con bajo contenido de estroncio y máxima velocidad de enfriamiento (A1), para confirmar los elementos presentes en las fases. La Fig. 4, muestra las fotomicrografías obtenida mediante Microscopía electrónica de barrido, de la zona estudiada, la figura superior esquematiza la zona estudiada, mientras que en las figuras de abajo, esquematizan la riqueza de los elementos señalados mediante las intensidad de las zonas claras color verde.

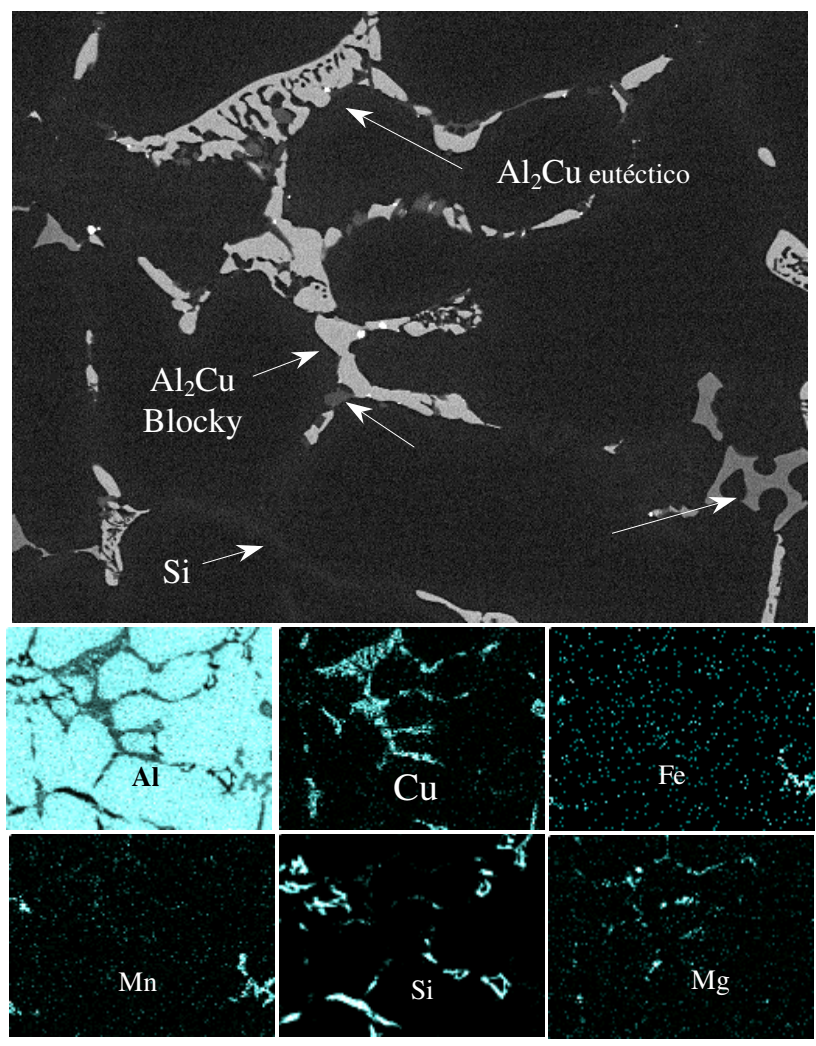

Fig. 4 Distribución química de los elementos mediante mapeo de rayos $\mathrm{x}$ por EDS, cualitativamente se determinan las fases de $\mathrm{Al}_{2} \mathrm{Cu}$ en forma 'Blocky" y eutectica, $\mathrm{Al}{ }_{15}(\mathrm{Fe}, \mathrm{Mn})_{3} \mathrm{Si}_{2}, \mathrm{Mg}_{2} \mathrm{Si}$, y $\mathrm{Si}$.

\section{CONCLUSIONES}

La Técnica de DTA es una alternativa viable para Análisis Térmico, debido a que se obtienen resultados en acuerdo a las otras técnicas con respecto a las reacciones principales.

El Estroncio afecta en forma secundaria la morfología de la interfase sólido - líquido, lo cual fue propuesto por Closset \& Gruzleski [3], [4], esto se corrobora mediante el análisis métalo gráfico ya que se observo 
un efecto muy marcado en la distribución de las fase menores hacia las zonas interdendríticas.

La presencia de un contenido elevado de estroncio disminuye la temperatura de solidificación del eutéctico de Si. El Estroncio favorece la formación de la fase $\mathrm{Al}_{2} \mathrm{Cu}$ con morfología tipo 'Blocky".

De acuerdo al análisis metalográfico realizado y los resultados del DTA, la tercera reacción corresponde a la descomposición de la fase rica en $\mathrm{Cu}$ con morfología 'Blocky" a eutéctico fino.

La baja velocidad de enfriamiento inhibe el efecto del estroncio sobre la morfología del Si eutéctico.

\section{AGRADECIMIENTOS}

Agradecemos, al COSNET su apoyo para el financiamiento de este trabajo. Al programa ECOS de SEP-CONACYT-ANUIES con el proyecto clave M99P04. Al Laboratorio de Materiales del INPENSIACET, CIRIMAT de Toulouse Francia, donde se realizó la experimentación.

\section{REFERENCIAS}

[1] M.M. Haque, M.A. Maleque; 'Effect of process variables on structure and properties of aluminum silicon piston alloy". Journal of Materials Processing Technology, 77, pp. 122-128. 1998.
[2] J.W Zindel, A.K. Dahle; 'Eutectic nucleation and growth in hypoeutectic Al-Si alloys at different strontium levels". Metallurgical and Materials Transactions, 32A, pp. 949-960. 2001

[3] J.E. Gruzleski; "The treatment of Liquid Aluminum-Silicon Alloys", American Foundrymens Society Inc.. 1990.

[4] B. Closset, J.E. Gruzleski; 'Microstuctures and thermal analysis of strontium treated aluminum alloys". American Foundrymens Society Inc. 1995.

[5] L. Backerud, G. Chai and J. Tamminen; 'Solidification characteristics of Aluminum Alloys", AFS/SKANALUMINIUM, Vol 2, pp. 71 229. 1990

[6] F.H. Samuel, H.W. Doty. Factors Controlling the Type and Morphology of $\mathrm{Cu}$-Containing Phases in 319 Al Alloy. AFS Transactions, 104, pp. 893-901. 1996.

[7] R. Colas, S. Valtierra, Spheroidization of the Al-Si Eutectic in a cast Aluminum alloy, Journal of materials engineering and performance, 9(6), pp. 616-622. 2000

[8] W. Kurtz, D.J. Fisher; 'Fundamentals of solidification". Third edition, trans tech publications, 1989.

[9] R. Luther, A. Oliver, F. J. Schutz; 'Métodos Numéricos ". Quinta Edición, 1985, pp 167-174. 